\title{
Caractérisation des filières bois-énergie et élaboration du schéma d'approvisionnement en bois-énergie de la région Analamanga, Madagascar
}

\section{Martial CHARPIN ${ }^{1}$ Denis LEgEAY2 \\ Njaratiana RABEMANANTSOA ${ }^{2}$ Frank RICHTER ${ }^{1}$}

${ }^{1}$ ECO Consulting Group GmbH \& Co. KG HersfelderStraße 17

36820 Oberaula

Allemagne

${ }^{2}$ Unité de Coordination du Programme d'appui à l'Agro-Sylviculture autour d'Antananarivo (UCP ASA)

Lot VA 28 FA Tsiadana

Antananarivo 101

Madagascar

\section{Auteur correspondant /}

Corresponding author:

Martial CHARPIN -

martial.charpin@eco-consult.com

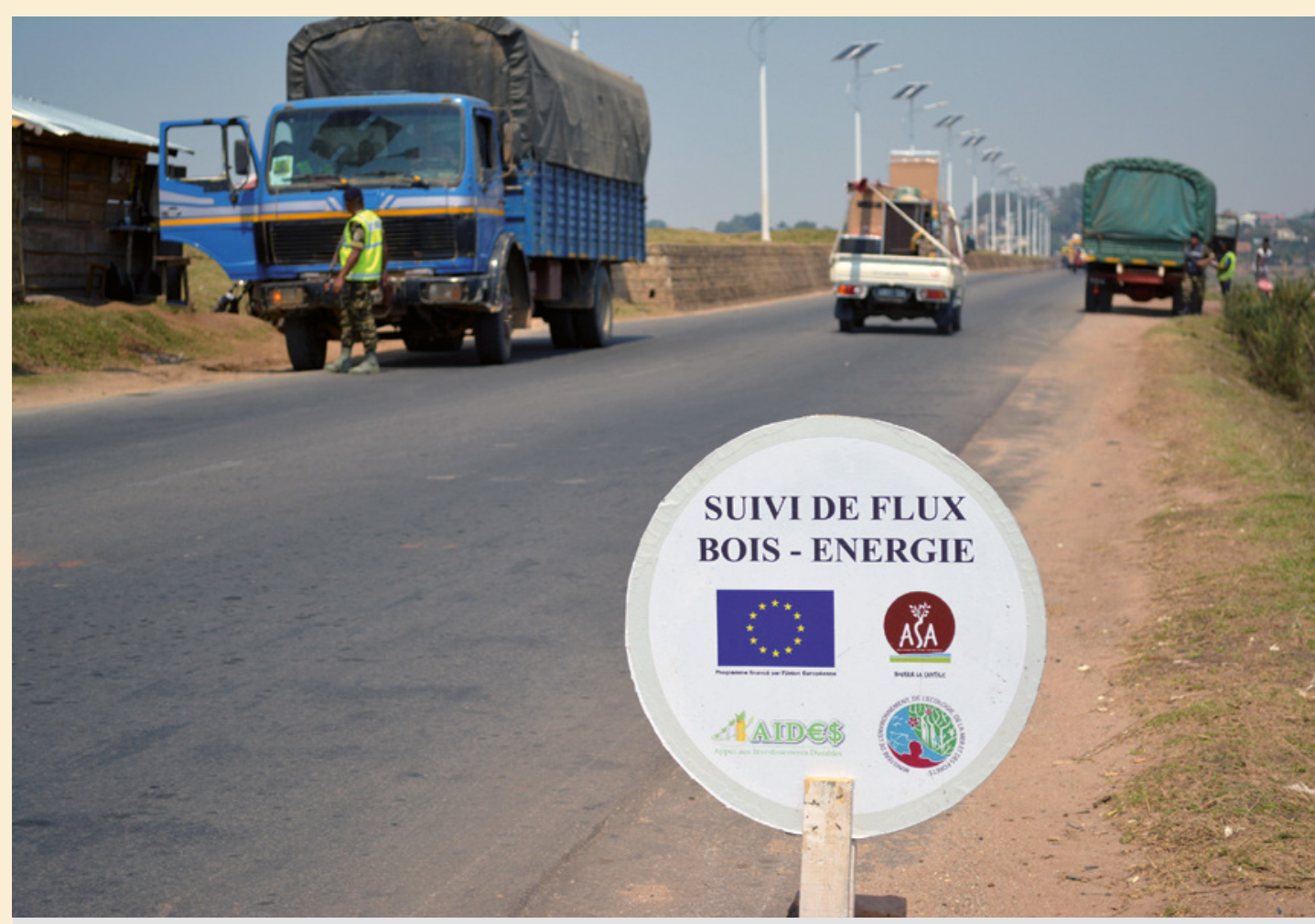

Photo 1.

Dispositif installé lors de l'évaluation des flux de charbon de bois à destination d'Antananarivo. Photo UCP ASA.

Doi : 10.19182/bft2019.340. a31704 - Droit d'auteur (C 2019, Bois et Forêts des Tropiques (C Cirad - Date de soumission : 18 juillet 2018 ; date d'acceptation : 23 janvier 2019 ; date de publication : $1^{\text {er }}$ avril 2019.

\section{cirad}

Citer l'article / To cite the article

Charpin M., Legeay D., Rabemanantsoa N., Richter F., 2019. Caractérisation des filières bois-énergie et élaboration du schéma d'approvisionnement en bois-énergie de la région Analamanga, Madagascar. Bois et Forêts des Tropiques, 340 : 13-25. Doi : https://doi.org/10.19182/bft2019.340. a31704 


\section{RÉSUMÉ}

\author{
Caractérisation des filières bois- \\ énergie et élaboration du schéma \\ d'approvisionnement en bois-énergie de \\ la région Analamanga, Madagascar
}

L'approvisionnement durable en combustibles domestiques est au centre des récentes politiques énergétiques et environnementales de l'État à Madagascar. La croissance constante de la demande en bois-énergie contraint les autorités publiques à l'action tant du point de vue national que local. Afin d'opérationnaliser ces orientations stratégiques, le premier Schéma régional d'approvisionnement en bois-énergie (SRABE) a été élaboré sous l'égide de la région Analamanga, en étroite collaboration avec les ministères responsables des forêts et de l'énergie. Réalisées dans le cadre du Programme d'appui à l'Agro-Sylviculture autour d'Antananarivo (ASA), financé par l'Union Européenne (10 $\mathrm{FED}$ ), plusieurs études majeures ont récemment été menées afin de disposer de données fiables et actualisées sur les filières bois-énergie. Les principaux résultats, présentés dans cet article, précisent l'ampleur de la demande en combustibles ligneux et les évolutions constatées sur les filières (bassin d'approvisionnement, stratégie commerciale, utilisation d'équipements de cuisson économes). Riches des expériences menées dans plusieurs régions du pays, les acteurs régionaux se sont engagés dans l'identification, la priorisation et la planification des actions ayant pour objectifs de sécuriser l'approvisionnement, d'accompagner la formalisation des acteurs économiques et de favoriser une utilisation plus efficiente des combustibles domestiques. Cette dynamique régionale positive n'est pourtant qu'une étape au regard de l'étendue du bassin d'approvisionnement de la capitale. L'enjeu est de parvenir rapidement à une intervention concertée sur le territoire d'au moins trois régions administratives.

Mots-clés : approvisionnement énergétique, filière bois-énergie, consommation domestique, utilisation des équipements de cuisson, Antananarivo, région Analamanga, Madagascar.

\section{ABSTRACT}

\author{
Characterisation of the fuelwood trade \\ and preparation of a fuelwood supply \\ plan for the Analamanga region in \\ Madagascar
}

Sustainable supplies of domestic fuel are a core issue addressed in recent government policies on energy and the environment adopted in Madagascar. Constantly increasing demand for fuelwood is forcing the public authorities to take action both nationally and locally. To bring their strategic guidelines into operation, the first regional fuelwood supply masterplan (SRABE) has been developed under the aegis of the Analamanga regional authority, in close collaboration with the Ministries responsible for forests and energy. Several major studies were recently conducted to collect reliable and current data on the fuelwood trade, carried out within the framework of the Agroforestry Support Programme around Antananarivo (ASA), financed by the European Union (10 ${ }^{\text {th }}$ EDF). The main results, presented in this article, describe the magnitude of demand for fuelwood and the trends noted in the different sectors (supply basin, commercial strategies, use of fuel-saving cookstoves). Building on a wealth of experience gained in several regions of Madagascar, regional players have begun to identify, prioritise and plan measures to keep supplies secure, help economic players integrate into the formal sector and promote more efficient use of domestic fuel. However, the emergence of these positive regional dynamics is only a step in the right direction given the size of the capital city's supply basin. The challenge is to rapidly achieve concerted action across at least three administrative regions.

Keywords: energy supplies, fuelwood sector, domestic consumption, use of cookstoves, Antananarivo, Analamanga region, Madagascar.

\section{RESUMEN}

\author{
Caracterización del sector de la \\ leña y elaboración del esquema de \\ aprovisionamiento en leña de la región \\ Analamanga, en Madagascar
}

El aprovisionamiento sostenible de combustibles domésticos es un aspecto central de las recientes políticas energéticas y medioambientales del estado de Madagascar. El crecimiento constante de la demanda de leña obliga a las autoridades a actuar tanto a nivel nacional como local. Para hacer operacionales estas orientaciones estratégicas, el primer esquema regional de aprovisionamiento de leña (SRABE) se ha elaborado al amparo de la región Analamanga, en estrecha colaboración con los ministerios responsables de los bosques y de la energía. Realizado en el marco del Programa de Apoyo Agroforestal alrededor de Antananarivo (ASA), financiado por la Unión Europea (10 $0^{\circ} \mathrm{FED}$ ), se han llevado a cabo varios estudios importantes recientemente para disponer de datos fiables y actualizados sobre la industria de la leña. Los principales resultados, presentados en este artículo, precisan la amplitud de su demanda de combustibles leñosos y la evolución constatada sobres los sectores (cuenca de aprovisionamiento, estrategia comercial, utilización de equipos de cocción económica). Con numerosas experiencias llevadas a cabo en varias regiones del país, los actores regionales se han comprometido a identificar, priorizar y planificar las acciones que tienen como objetivo asegurar el aprovisionamiento, acompañar la formalización de los actores económicos y favorecer una utilización más eficiente de los combustibles domésticos. Esta dinámica regional positiva solo es, sin embargo, una etapa con respecto a la extensión de la cuenca de aprovisionamiento de la capital. La apuesta es alcanzar rápidamente una intervención concertada en el territorio de al menos tres regiones administrativas.

Palabras clave: aprovisionamiento energético, sector de la leña, consumo doméstico, utilización de equipamientos de cocción, Antananarivo, región Analamanga, Madagascar. 


\section{Introduction}

Source d'énergie abordable, le bois fait partie du quotidien de tous les Malgaches, qu'ils soient urbains ou ruraux. Plus de $90 \%$ des ménages l'utilisent pour la cuisson des aliments sous forme de bois de feu et/ou de charbon de bois. La prépondérance du bois-énergie dans le bouquet énergétique national s'explique, en partie, par le manque d'attractivité, de compétitivité et d'accessibilité des alternatives énergétiques, gaz, électricité, briquettes ou éthanol combustible. Pourtant, le bois-énergie a longtemps été dénigré par les décideurs et gaspillé par les utilisateurs. Considéré comme archaïque et salissant, son prix modique ne reflète pas les coûts réels qui devraient être associés à une gestion durable des ressources forestières. Le faible pouvoir d'achat, voire l'extrême pauvreté d'une part importante de la population malgache, participent au maintien des prix de vente bas aux consommateurs.

Force est de reconnaître que l'intervention publique est délicate et périlleuse lorsqu'il s'agit d'organiser cette filière stratégique. Les initiatives de régulation et de contrôle des flux de bois-énergie, orchestrées par les autorités administratives et les services techniques compétents, sont rendues difficiles du fait des risques de mouvements sociaux qu'elles peuvent engendrer. De plus, la diversité des acteurs en présence complexifie l'intervention, du fait des intérêts divergents et parfois contradictoires qu'ils défendent.

Ces difficultés ne doivent toutefois pas être un frein à l'action publique. La valorisation énergétique de la biomasse ligneuse est un sujet particulièrement intéressant pour porter des réflexions et des actions interministérielles, entre les ministères en charge de l'énergie et des forêts, bien sûr, mais également avec les ministères en charge de la décentralisation, du foncier, du commerce et de l'aménagement du territoire. Conscientes de ces enjeux, les autorités malgaches ont récemment formulé et adopté la Stratégie nationale d'approvisionnement en bois-énergie (SNABE). Déclinaison intersectorielle de la Nouvelle Politique de l'énergie et de la Nouvelle Politique forestière, la SNABE recommande d'élaborer des documents opérationnels au niveau régional devant préciser les mesures à prendre, ainsi que les activités à mener dans le but de rétablir le bilan offre/demande en bois-énergie (Sepp, 2013). La production de données actualisées permettant de caractériser la situation actuelle sur les filières bois-énergie dans la région Analamanga s'inscrit dans cette recommandation. Sur la base du diagnostic présenté dans le présent article, un processus participatif et itératif réunissant les différents groupes d'acteurs membres de la plateforme régionale biomasse-énergie a été engagé au cours de l'année 2018, dans le but d'élaborer un schéma régional d'approvisionnement en bois-énergie pour la région Analamanga.

\section{Méthodologie}

\section{Sites d'étude}

L'analyse de la filière bois-énergie concerne la région administrative d'Analamanga. Située dans la zone géographique des Hautes Terres, la région Analamanga s'étend sur environ $3 \%$ du territoire national $\left(17442 \mathrm{~km}^{2}\right)$ et abrite la capitale malgache, Antananarivo. Près de $18 \%$ de la population nationale, soit environ 4,6 millions d'habitants, vivent dans la région. Elle se démarque des autres régions du pays par son fort taux d'urbanisation (69\%) et une densité de population relativement élevée (264 hab. $/ \mathrm{km}^{2}$ ).

\section{Collecte des données}

Les données disponibles pour caractériser les filières d'approvisionnement en bois-énergie de la région Analamanga, et plus particulièrement de l'agglomération d'Antananarivo, étaient partielles ou obsolètes. Les dernières données de référence avaient été produites au cours des années 1990 (Ramamonjisoa, 1993 ; UPED, 1994). Par conséquent, des études complémentaires étaient nécessaires, notamment pour caractériser les flux de bois-énergie à destination de l'agglomération d'Antananarivo, mais aussi la structure des prix et le potentiel de production présent sur le territoire de la région Analamanga.

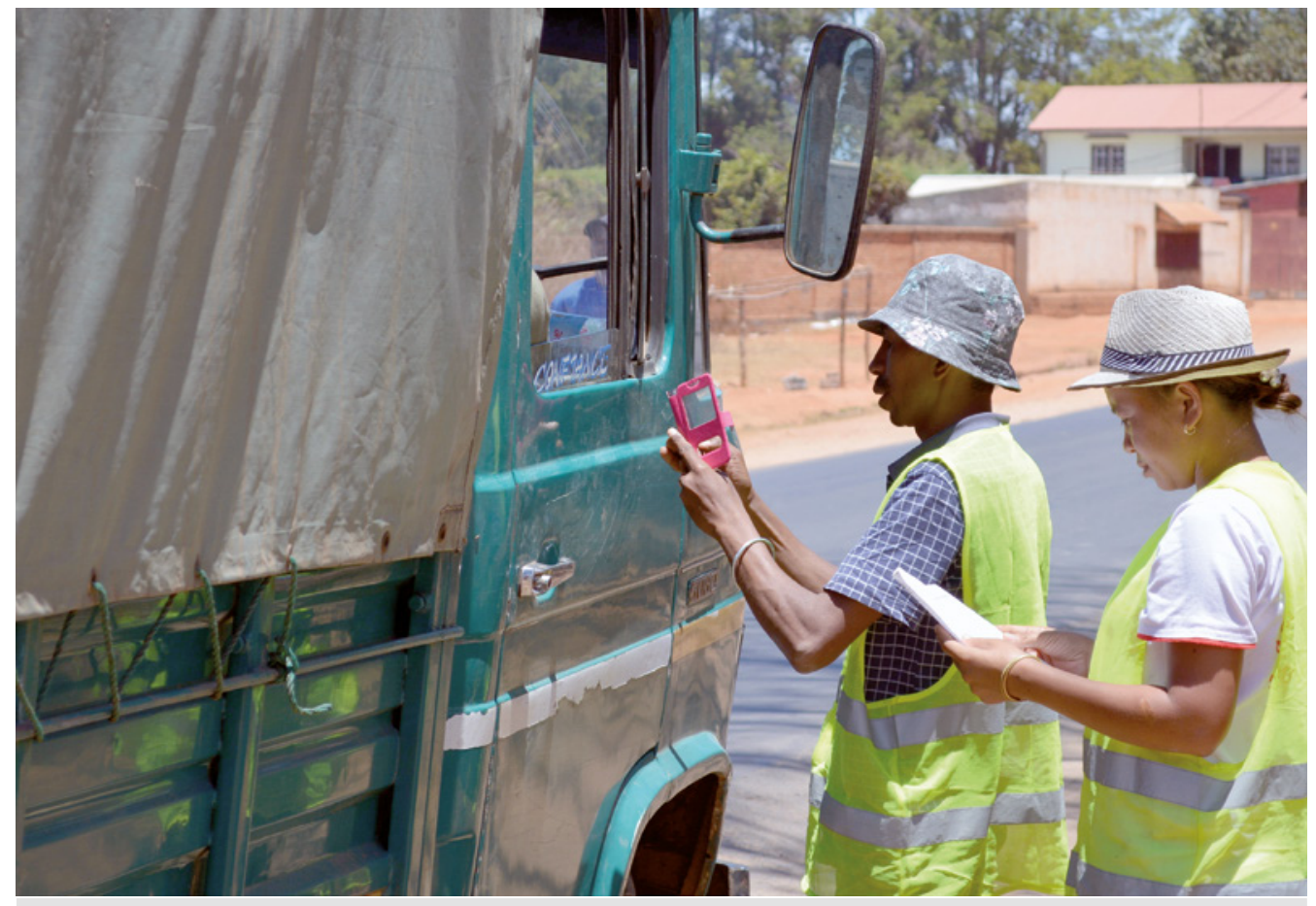

Photo 2.

Enquêteurs interrogeant un transporteur de charbon de bois à l'entrée de l'agglomération d'Antananarivo.

Photo UCP ASA. 


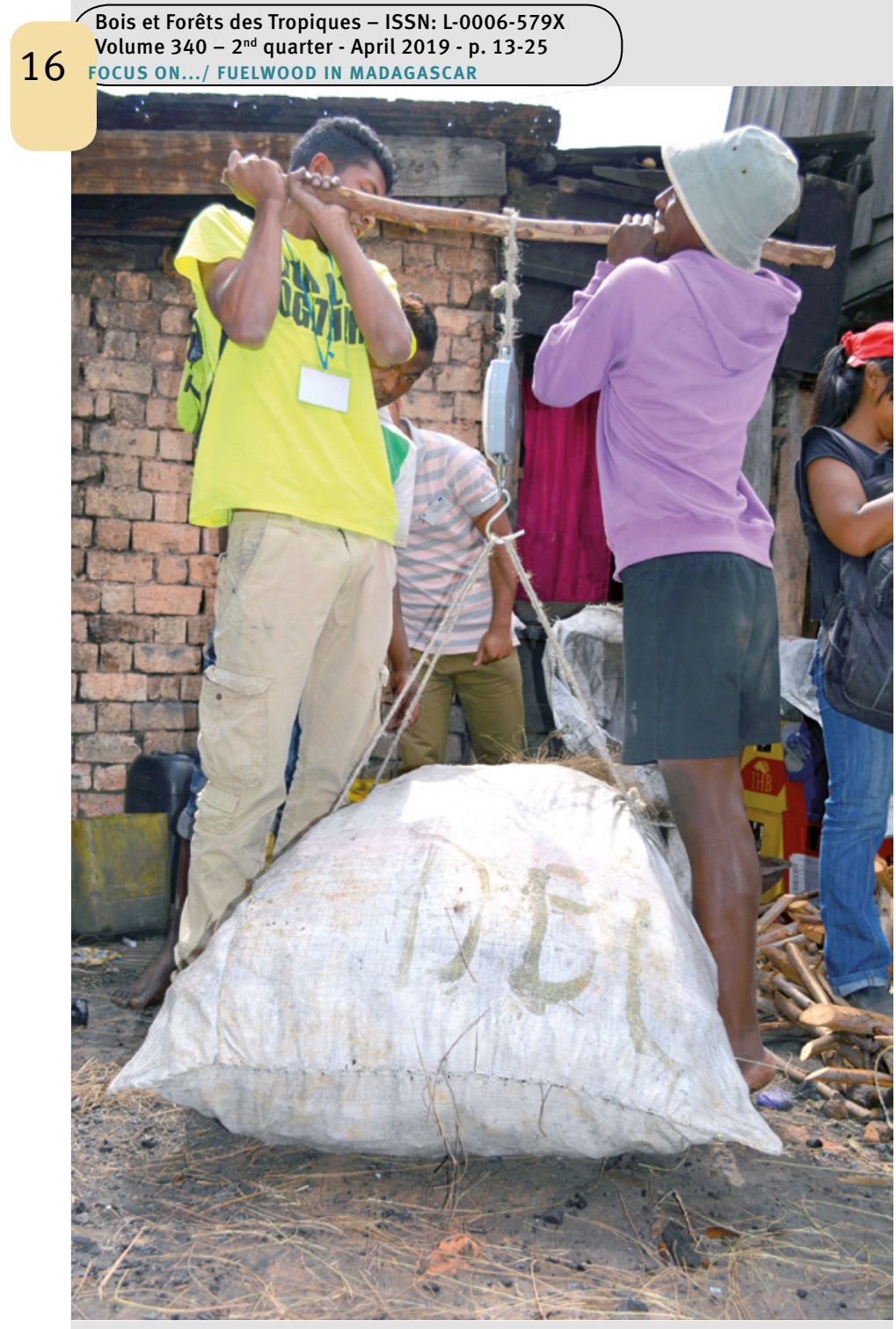

Photo 3.

Pesée des sacs de charbon de bois approvisionnant Antananarivo.

Photo UCP ASA.

Afin de disposer de ces données actualisées, quatre études majeures ont récemment été engagées et finalisées dans le cadre du Programme d'appui à l'Agro-Sylviculture autour d'Antananarivo (ASA), financé par l'Union Européenne (10 FED). Elles ont été commanditées et suivies par l'unité de coordination du programme dont l'assistance technique est assurée par le consortium IRAM-ECO Consulting-BEST. II s'agit de :

- L'étude des flux de bois-énergie entrant dans la ville d'Antananarivo (AIDES, 2018a). Un dispositif routier a été installé d'octobre 2016 à juillet 2017 afin d'évaluer les quantités de bois de feu et de charbon de bois transportées à destination de l'agglomération d'Antananarivo. Basée sur cinq postes de contrôle situés sur les principaux axes routiers convergeant vers la capitale, la collecte de données a considéré trois campagnes réparties sur l'année : saison sèche (octobre), saison des pluies (mars) et contre-saison agricole (juin).
- L'étude du potentiel de production en bois-énergie dans le bassin d'approvisionnement de la ville d'Antananarivo (Land Ressources, 2018). Sur la base des résultats de l'étude précédente, une évaluation du capital forestier sur pied a été réalisée en adoptant une méthodologie basée sur les traitements d'images satellitaires (par télédétection) et l'organisation d'un inventaire forestier afin de disposer des informations sur l'occupation du sol et les volumes réels sur pied.

- L'étude des prix du bois-énergie dans la ville d'Antananarivo (AIDES, 2018b). Des enquêtes portant sur les prix du bois-énergie et des autres combustibles de cuisson ont été menées entre juillet 2017 et mai 2018. Les points de vente suivis étaient répartis sur l'ensemble de l'agglomération d'Antananarivo afin de considérer les quartiers populaires, résidentiels, les places de marché et les communes périphériques.

- L'étude de marché et l'analyse technico-économique de la filière "foyers améliorés » dans la région (LLD, 2016). Des enquêtes ont été menées auprès des ménages et des artisans producteurs de foyers améliorés situés dans les districts de Anjozorobe, Manjakandriana, Arivonimamo, Andramasina, Atsimondrano, Avaradrano et Ankazobe.

Les principales données quantitatives et qualitatives collectées ou produites lors de ces études permettent de caractériser la situation actuelle de la filière bois-énergie. Ce travail s'inscrivait dans le processus d'élaboration du schéma régional d'approvisionnement en bois-énergie de la région Analamanga (photos 1, 2 et 3 ).

\section{Résultats}

\section{Un capital forestier régional éprouvé}

Les forêts naturelles ne couvrent plus qu'une infime partie du territoire de la région. Les superficies des catégories "forêt dense humide " et "forêt humide dégradée " représentent respectivement 13738 ha et 38604 ha. Ces espaces boisés sont majoritairement " protégés » dans le cadre de leur classement en aires protégées (catégorie IV et $\mathrm{V}$ ) ou de transferts de gestion entre l'État et des tiers (gestion contractuelle des forêts et gestion locale sécurisée). La couverture forestière est largement dominée par les « savanes arborée et arbustive » qui s'étendent sur 474596 ha, soit $62,5 \%$ de la couverture forestière. L'analyse de l'évolution des superficies forestières sur la période 2005-2013 (MEEF-ONE, 2015) révèle des taux annuels de réduction du couvert forestier de 0,22 \% pour la période 2005-2010 et de $0,32 \%$ pour la période 2010-2013. En conséquence, bien que les superficies forestières soient déjà réduites, une augmentation de la déforestation sur le territoire régional s'est manifestée au cours des dernières années.

Hormis les forêts naturelles, la superficie des terres boisées comprend également les plantations forestières. Le genre Eucalyptus, introduit entre 1857 et 1889 à Madagascar, a rapidement été adopté par l'administration forestière, puis par la population rurale, en particulier sur les Hautes Terres (Bertrand, 1999). La plantation forestière s'est pro- 
Tableau I.

Superficie par strate forestière et type d'arbres hors forêts (année 2018).

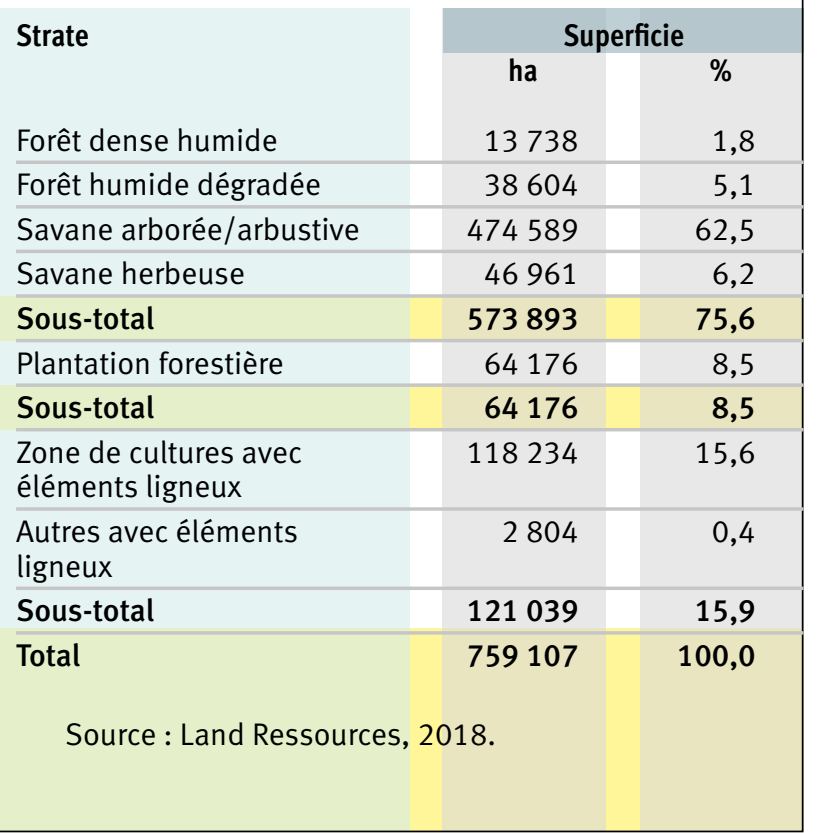

gressivement intégrée dans les stratégies paysannes de mise en valeur des terres et de création de revenus (photo 4).

La superficie exacte des plantations forestières fait débat. Au cours des cinq dernières années, plusieurs études ont fourni des valeurs diverses variant de 64176 à plus de 90000 ha (Ramamonjisoa et al., 2016 ; Land Ressources, 2018). Dans le cadre de ces travaux, la valeur basse a été retenue. Enfin, la troisième catégorie considérée pour l'estimation de l'offre de bois concerne l'« arbre hors forêt/espace agroforestier ». Elle couvre une superficie de 121039 ha. Le tableau I présente la synthèse des superficies par catégorie.

En considérant les taux de prélèvement et les rotations précisés dans le tableau II, l'offre potentielle théorique en bois-énergie provenant de l'exploitation raisonnée des arbres situés dans les espaces forestiers et agroforestiers de la région Analamanga est estimée à $604447 \mathrm{~m}^{3}$ par an (tableau II). En considérant une équivalence de $700 \mathrm{~kg} / \mathrm{m}^{3}$, l'offre potentielle théorique est estimée à 423113 tonnes de bois (Richter et Charpin, 2018). Selon les résultats de l'enquête-flux, 105604 tonnes de charbon équivalentes à 880033 tonnes de bois proviennent de la région Analamanga et sont transportées à destination de la capitale. Les prélèvements réels sont donc au moins deux fois supérieurs aux capacités de production des espaces forestiers de la région.

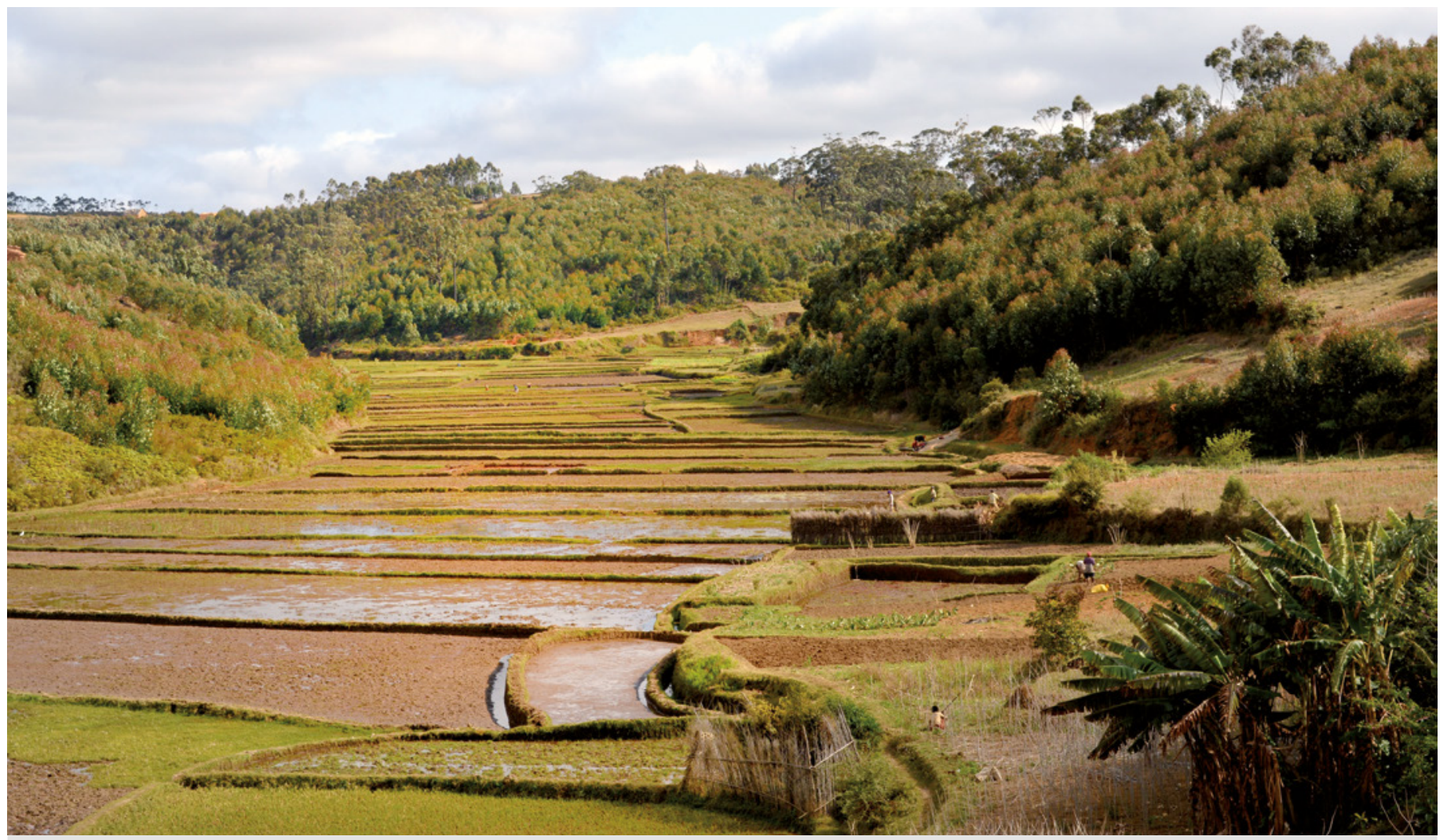

Photo 4.

Intégration des plantations d'eucalyptus dans les stratégies paysannes de mise en valeur des terres, district de Manjakandriana.

Photo UCP ASA. 
Tableau II.

Répartition de l'offre totale en bois-énergie dans la région Analamanga (année 2018).

Source de bois-énergie

\begin{tabular}{|c|c|c|}
\hline $\begin{array}{l}\text { Production des forêts } \\
\text { naturelles }^{2}\end{array}$ & $\begin{array}{c}m^{3} / a^{1} \\
149507\end{array}$ & $\begin{array}{l}\text { t bois/an } \\
104655\end{array}$ \\
\hline $\begin{array}{l}\text { Production des planta- } \\
\text { tions forestières } \\
\text { ti }\end{array}$ & 319149 & 223404 \\
\hline Arbres hors forêts ${ }^{4}$ & 135791 & 95054 \\
\hline Total exploité & 604447 & 423113 \\
\hline
\end{tabular}

Source : Richter et Charpin, 2018.

${ }^{1}$ Équivalent en $\mathrm{m}^{3}$ calculé avec un rendement pondéral de la carbonisation fixé à $12 \%$ et une densité du bois de $0,7 \mathrm{t} / \mathrm{m}^{3}$. ${ }^{2}$ Volume de bois-énergie potentiellement exploitable calculé avec : volume sur pied ( $\left.\mathrm{m}^{3} / \mathrm{ha}\right) \mathrm{x}$ taux d'exploitation / rotation. Taux d'exploitation $=50 \%$, rotation : forêt dense humide $=30$ ans ; forêt humide dégradée $=20$ ans ; savane arborée/arbustive $=6$ ans. ${ }^{3}$ Taux d'exploitation $=100 \%$; rotation $=6$ ans.

${ }^{4}$ Taux d'exploitation $=75 \%$; rotation $=7$ ans.

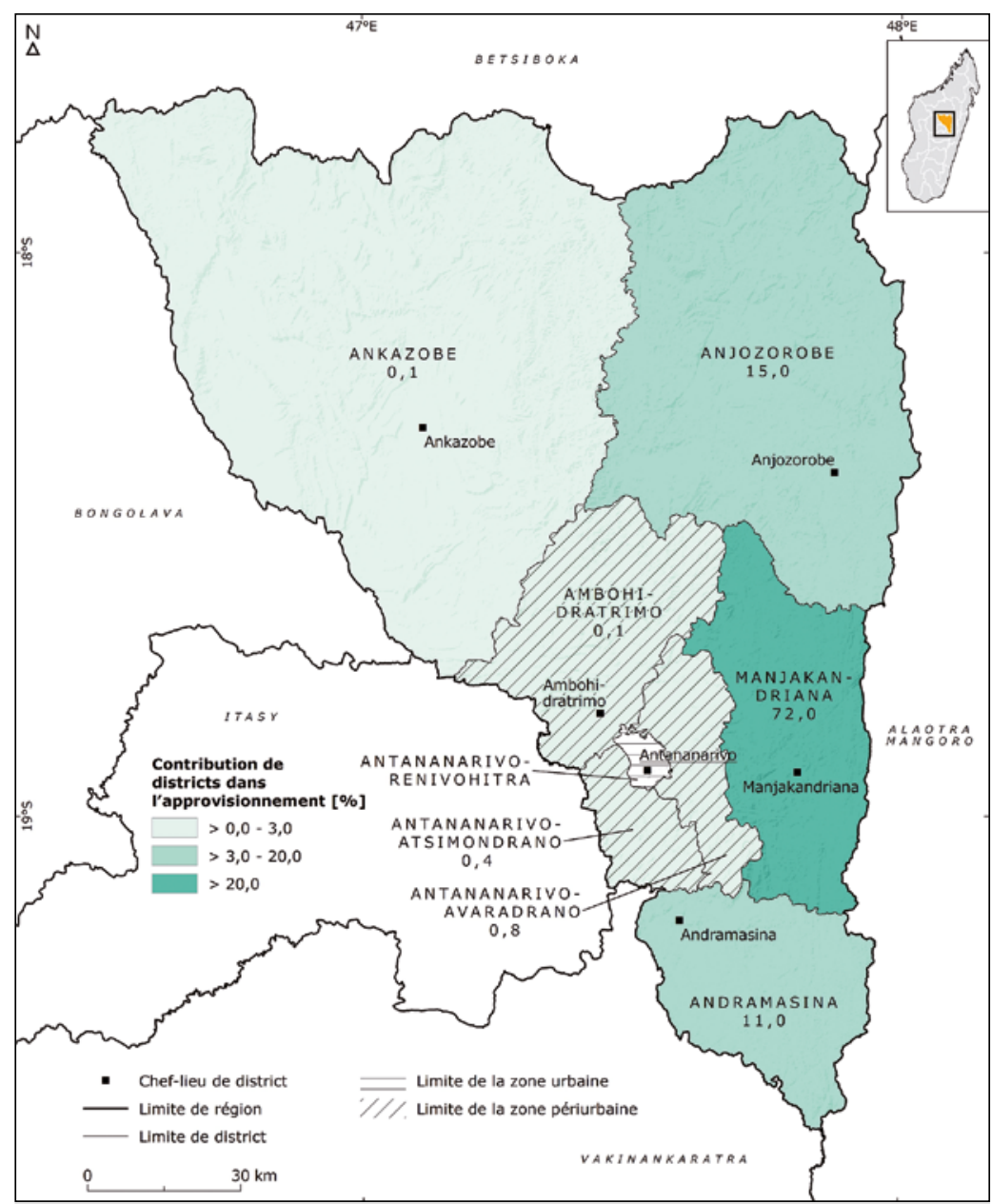

Figure 1.

Contribution annuelle des principaux districts de la région Analamanga dans l'approvisionnement en charbon de la ville d'Antananarivo (année 2017).

\section{Tableau III.}

Provenance du charbon de bois approvisionnant la ville d'Antananarivo par district dans la région Analamanga (année 2017).

\begin{tabular}{l|r|r|}
\hline $\begin{array}{l}\text { District } \\
\text { (nom) }\end{array}$ & \multicolumn{2}{c}{ Quantité de charbon } \\
\hline t/an & \multicolumn{1}{c}{$\%$} \\
\hline Manjakandriana & 76381 & 72,0 \\
\hline Anjozorobe & 15685 & 15,0 \\
\hline Andramasina & 11933 & 11,0 \\
\hline Antananarivo Avaradrano & 861 & 0,8 \\
\hline Antananarivo Atsimondrano & 452 & 0,4 \\
\hline Ankazobe & 153 & 0,1 \\
\hline Ambohidratrimo & 140 & 0,1 \\
\hline Total & 105604 & 100,0
\end{tabular}

Source : AIDES, $2018 \mathrm{a}$.

Les flux de bois-énergie constatés en 2016 et 2017 permettent de préciser les districts où se concentre l'exploitation forestière. Le district fournissant actuellement les volumes de charbon les plus élevés est Manjakandriana, avec 76381 tonnes de charbon expédiées vers la capitale, ce qui représente $72 \%$ des flux provenant de la région Analamanga (tableau III). Ce taux s'explique par l'importance des plantations forestières privées dans ce district. Deux autres districts, Anjozorobe et Andramasina, fournissent également une part significative de l'approvisionnement en bois à vocation énergétique, avec respectivement $15 \%$ et $11 \%$ des flux régionaux (figure 1). Certains districts tel Antananarivo Avaradrano étaient pourtant des lieux d'approvisionnement relativement importants dans les années 1990 (UPED, 1994). Actuellement, la contribution de ce district à l'approvisionnement de la capitale est marginale ( $1 \%)$.

Toutes provenances confondues, les essences forestières majoritairement exploitées pour la production de charbon de bois sont les eucalyptus (74\%), provenant principalement de reboisements privés, et les pins ( $22 \%$ ), provenant principalement des rebus d'exploitation de la société Fanalamanga située dans la région d'Alaotra Mangoro. La part des essences provenant des forêts naturelles s'élève à $4 \%$ (figure 2). Ce taux pourrait toutefois s'avérer sous-estimé puisque ces résultats se basent sur la déclaration des transporteurs sans vérification systématique de leur chargement. 


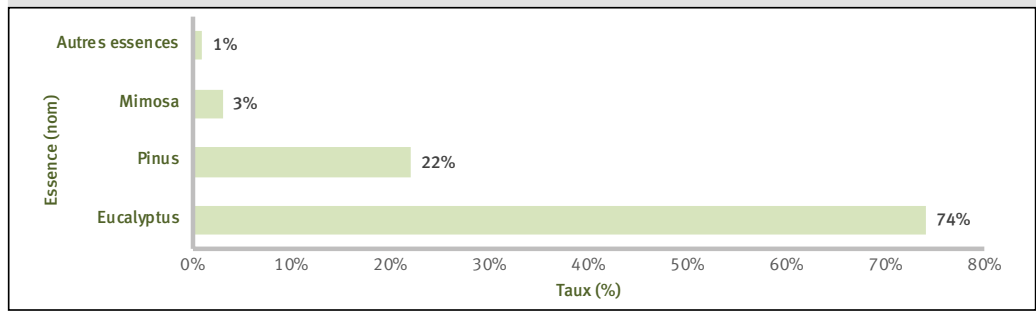

Figure 2.

Répartition par essence des quantités de charbon à destination d'Antananarivo (année 2017). Source : FTM BD 100/BD 200. de l'agglomération d'Antananarivo, les résultats présentent au contraire des flux de combustibles plus importants au cours de la saison des pluies et de la contre-saison agricole, avec respectivement 18161 tonnes par mois et 22182 tonnes par mois (figure 4).

Deux éléments principaux expliquent ces résultats. Tout d'abord, l'importance des flux au cours de la saison des pluies ne signifie pas que la production de charbon de bois est plus importante pendant cette période. L'abattage et la carboni-

\section{Un bassin d'approvisionnement toujours plus grand}

La demande en bois-énergie de la population régionale, en particulier de la grande agglomération d'Antananarivo (GAA), ne peut être satisfaite par les seules ressources forestières situées sur le territoire de la région Analamanga. Ce constat est connu puisque des sources d'approvisionnement situées hors de la région étaient d'ores et déjà documentées dans les années 1990 (Ramamonjisoa, 1991 ; UPED, 1994). La dernière enquête-flux confirme cet état de fait et renseigne sur l'étendue des prélèvements hors de la région Analamanga.

L'enquête a permis de recenser 396 communes contribuant à l'approvisionnement de la capitale, réparties dans 71 districts relevant de 19 régions du pays (figure 3).

Les quantités de charbon provenant des régions hors Analamanga et à destination de la GAA atteignent près de 125000 tonnes de charbon de bois, équivalant à près de 1,5 million de mètres cubes de bois. Avec respectivement $44 \%$ et $5 \%$, les régions de Alaotra Mangoro et Vakinankaratra fournissent la moitié des volumes recensés. Les prélèvements en provenance de ces régions se sont intensifiés au cours des quinze dernières années. Pour la région d'Alaotra Mangoro, le taux annuel de déforestation est passé de 1,72\% pour la période 2005-2010 à 2,14 \% pour la période 2010-2013. Ce taux est l'un des plus élevés du pays (MEEFONE, 2015).

L'enquête-flux et le suivi des prix de vente du charbon ont permis de mettre en lumière les stratégies commerciales de certains acteurs économiques intervenant dans la filière charbon de bois. Il est généralement convenu que les flux de charbon sont plus importants en saison sèche. Les conditions météorologiques ainsi que le calendrier agricole sont des facteurs favorables à l'organisation de l'exploitation forestière. Or, dans le cadre de l'étude de l'approvisionnement

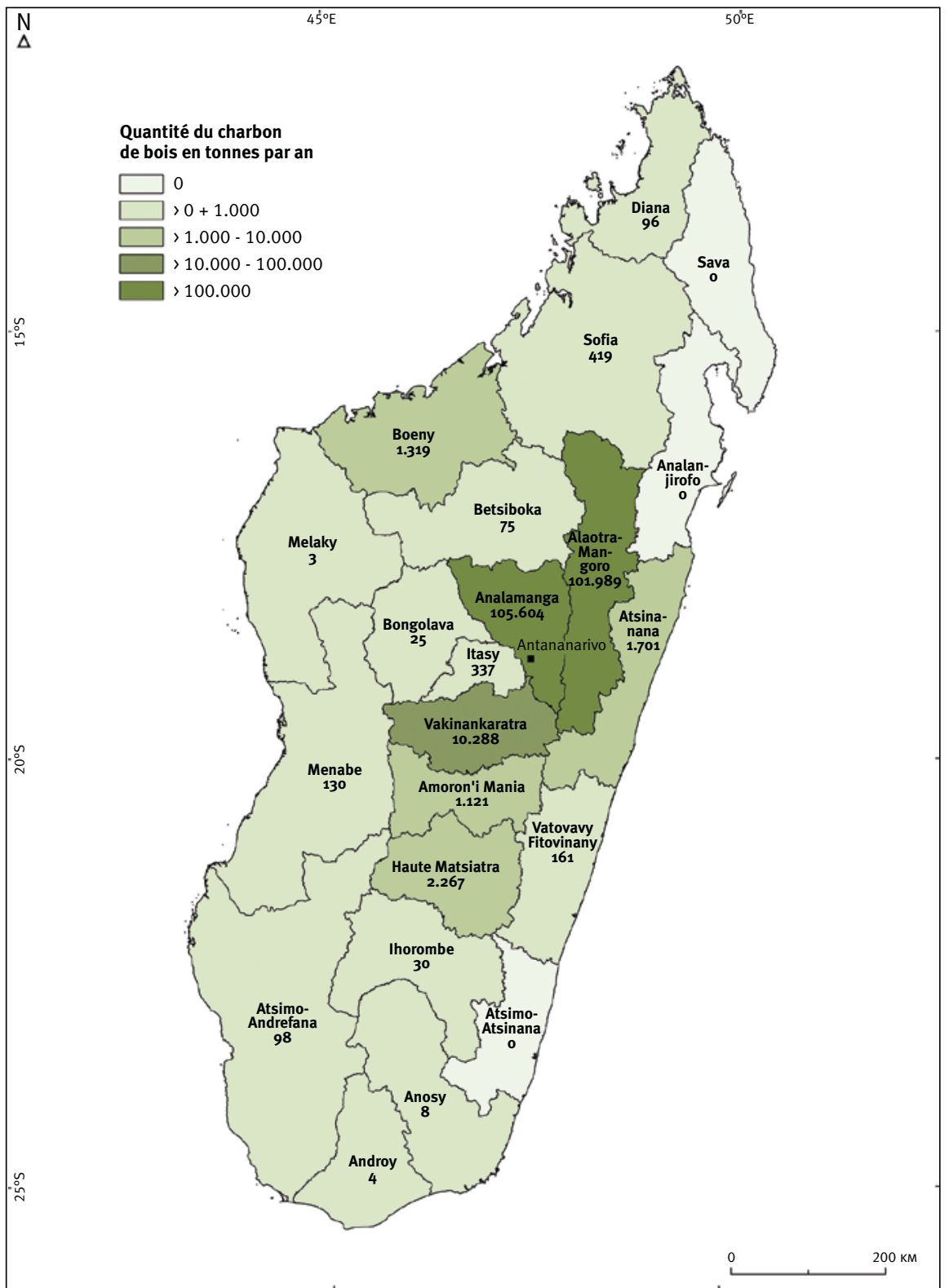

Figure 3.

Contribution annuelle des régions limitrophes à l'approvisionnement en charbon de l'agglomération d'Antananarivo (année 2017). Source : FTM BD 100/BD 200. 


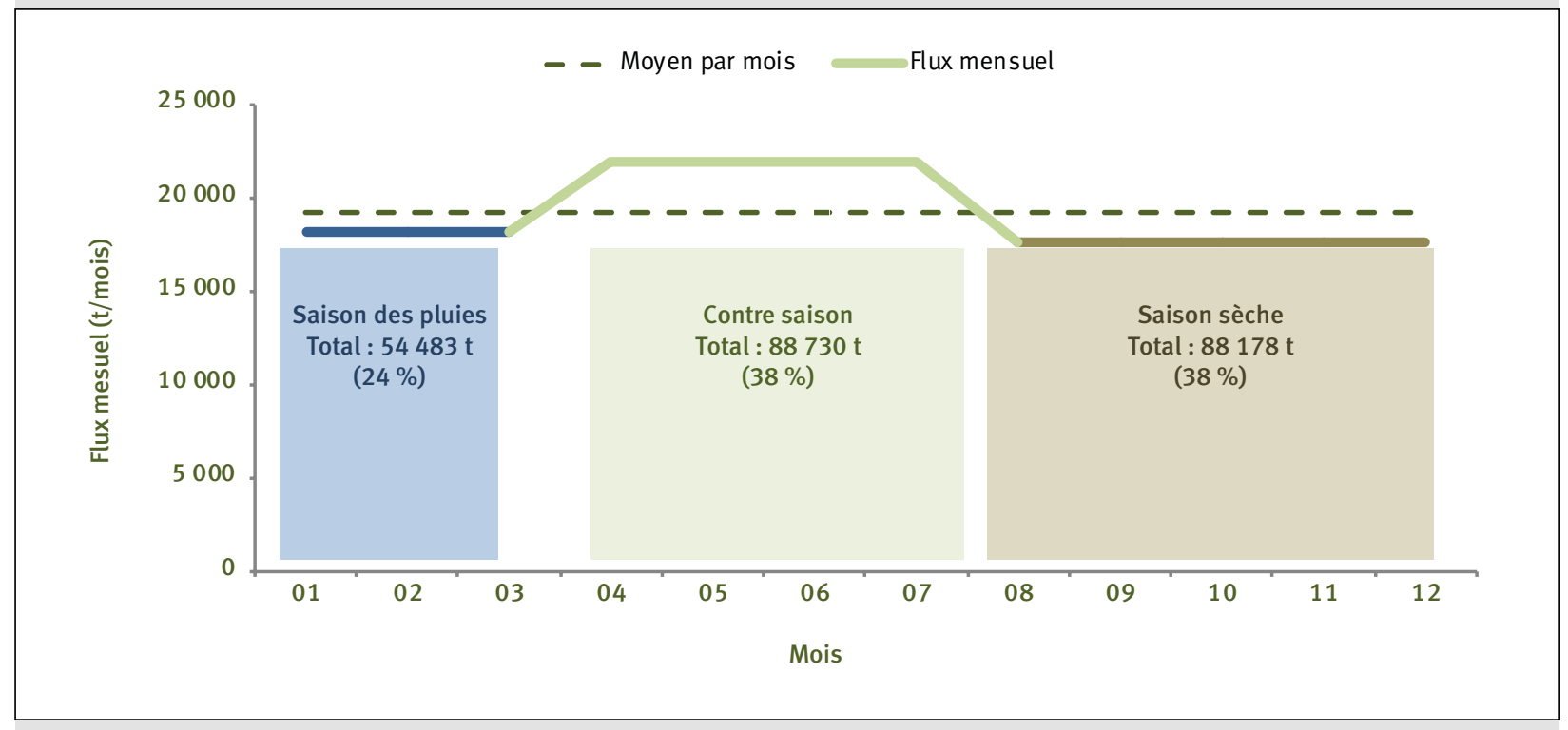

Figure 4.

Flux mensuels de charbon de bois à destination d’Antananarivo (année 2017).

disposant d'une assise financière suffisante ou de sources de revenus diversifiées leur permettant d'organiser le transport des zones d'exploitation vers une localité située à proximité d'un axe routier praticable et de stocker le charbon avant de l'écouler au cours de la saison des pluies. Cette organisation permet également de sécuriser l'approvisionnement de la capitale en compensant la réduction des flux provenant des zones inaccessibles pendant la saison des pluies.

Second constat, au cours de la contre-saison agricole, les conditions climatiques s'améliorent et permettent aux paysans d'organiser des chantiers de carbonisation. À l'approche des fêtes de l'Indépendance et des évènements socioculturels associés à cette période (retournement des morts, notamment), les paysans charbonniers s'orientent vers l'exploitation forestière pour générer rapidement des revenus et couvrir leurs dépenses. Ces différents opérateurs peuvent ainsi profiter de l'augmentation constatée des prix du charbon de janvier à avril. La figure 5 présente l'évolution du prix du charbon au détail et en sac.

\section{Le bois-énergie, un produit de première nécessité}

La quasi-totalité des ménages de la région Analamanga utilisent les combustibles ligneux ( $97 \%$ ). Malgré la présence d'autres sources énergétiques comme l'électricité, le gaz ou les briquettes, la part des combustibles de substitution au bois-énergie reste faible (Rafitoson, 2017). Cette situation s'explique en grande partie par les prix élevés des énergies dites « modernes » et les habitudes culinaires des ménages. La figure 6 présente les taux d'utilisation de ces différentes sources d'énergie.

Les consommations annuelles moyennes par habitant en milieu urbain sont évaluées à près de $104 \mathrm{~kg}$ de charbon de bois ou $270 \mathrm{~kg}$ de bois de feu. Les ménages ruraux consomment annuellement $513 \mathrm{~kg}$ de bois de feu ou $91 \mathrm{~kg}$ de charbon de bois par personne (tableau IV). En comparaison avec les consommations constatées dans d'autres régions du pays, telles que Diana ou Boeny, ces valeurs sont plus faibles. Ce constat peut s'expliquer par une recherche d'économie de combustibles du fait de leur coût relativement élevé pour les ménages et également par une modification des habitudes des consommateurs. Du fait de l'urbanisation, des horaires de travail et des contraintes de déplacements dans l'agglomération, les habitudes des consommateurs évoluent. Lors du déjeuner, voire du dîner, les habitants de la capitale privilégient la restauration de rue plutôt que la préparation de repas à domicile. Là encore, la source d'énergie principalement utilisée est le bois. 


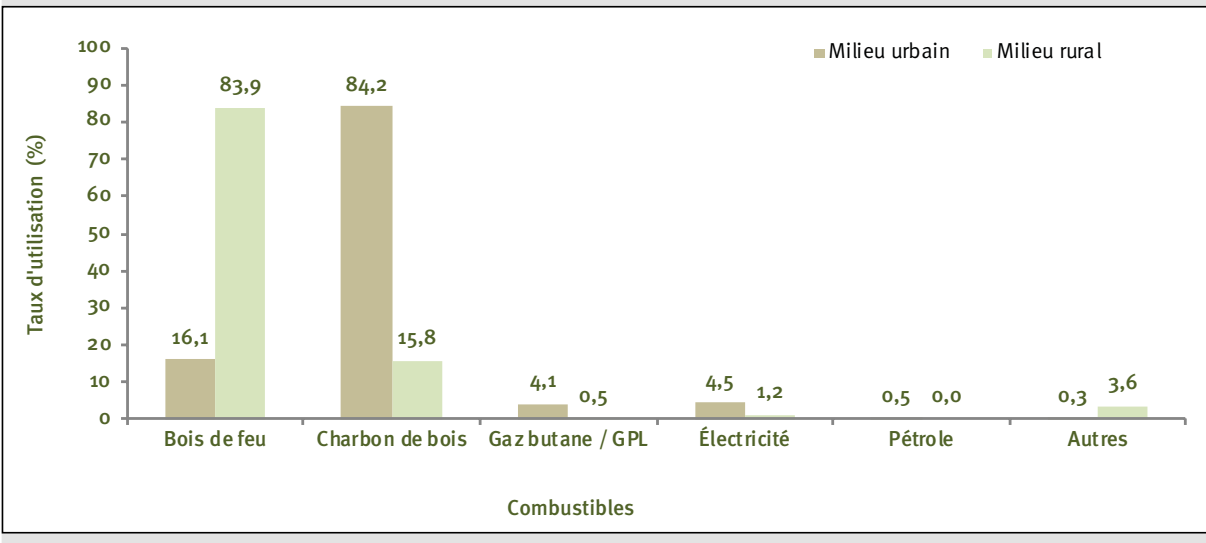

Figure 6.

Importance relative des principaux combustibles domestiques en milieu urbain et rural (année 2016).

\section{Le charbon de bois, premier combustible domestique de la région}

En 2018, la population de la région Analamanga est estimée à 4586864 habitants dont 69,3\% vivent en milieu urbain et $30,7 \%$ en milieu rural. La région concentre donc près de $18 \%$ de la population malgache sur moins de $3 \%$ du territoire national. En se basant sur les chiffres de consommation présentés précédemment, la consommation de charbon de bois des ménages de la région Analamanga s'élève à 376063 tonnes de charbon de bois. Environ 268380 tonnes de charbon de bois sont consommées dans les centres urbains de la région, dont 230392 tonnes pour la seule agglomération d'Antananarivo. En considérant la prédominance des techniques traditionnelles de carbonisation, cette demande régionale en charbon de bois correspond à un volume de $4829297 \mathrm{~m}^{3}$ de bois (tableau IV). À titre de comparaison, le volume de bois transformé en charbon chaque année sur la Grande Île est estimé à 6,3 millions de mètres cubes (MEH, 2015). La seule population présente dans la région Analamanga consomme donc près des trois quarts de la production nationale de charbon de bois.

Si l'on se réfère à l'estimation de la production durable de bois-énergie fournie par les espaces forestiers de la région $\left(604447 \mathrm{~m}^{3}\right)$, seul un huitième de la demande domestique pourrait être couvert.

Tableau IV.

Consommation annuelle des ménages urbains et ruraux en bois-énergie - Région Analamanga (année 2018).

\begin{tabular}{|c|c|c|c|}
\hline \multirow[t]{2}{*}{ Combustible } & \multicolumn{3}{|c|}{ Milieu } \\
\hline & Urbain (t/an) & Rural (t/an) & Total (t/an) \\
\hline Bois de feu & 133106 & 113546 & 246652 \\
\hline Charbon de bois & 268381 & 107681 & 376063 \\
\hline Équivalent en $\mathrm{m}^{3}$ /an de bois* & 3385169 & 1444128 & 4829297 \\
\hline
\end{tabular}

\section{Une utilisation progressive des foyers améliorés}

En milieu rural, la grande majorité des ménages utilisent des foyers traditionnels à bois. Les taux de pénétration des foyers améliorés par commune rurale oscillent entre 0,5 et $5,7 \%$ (LLD, 2016). En revanche, les ménages urbains s'équipent progressivement de foyers améliorés. En 2016, le taux de pénétration des foyers améliorés dans les communes urbaines était évalué à $61 \%$. Parmi ces ménages, 36,6\% utilisent de façon combinée le foyer amélioré et le foyer traditionnel afin d'adapter l'équipement de cuisson au type d'aliment à cuire. Les modèles de foyer amélioré produits dans l'agglomération d'Antananarivo sont principalement :

- les foyers cylindriques avec coque métallique ;

- les foyers coniques avec coque métallique ;

- les foyers sans coque.

Les foyers à charbon de forme cylindrique avec coque métallique représentent $69,5 \%$ de la production régionale (tableau V). Selon les producteurs, ce modèle de foyer est le plus facile à confectionner, du fait de sa forme géométrique constituée d'une seule partie. La durée de fabrication se trouve ainsi réduite.

Plus d'une centaine de familles d'artisans s'activent dans la confection de foyers améliorés avec insert céramique. La production mensuelle par famille varie entre 100 et 360 foyers selon l'expérience, la spécialisation et la localisation géographique de la famille. Les plus performantes se concentrent dans le district d'Antananarivo Atsimondrano. En considérant les moyens de production des artisans et la main-d'œuvre disponible auprès de chaque atelier, la capacité de production maximale est estimée à près de 28000 foyers améliorés.

En 2016, le volume mensuel des ventes de foyers améliorés était évalué à 18680 unités (LLD, 2016).

\section{Discussion}

Dans le contexte socio-économique actuel, la part des combustibles ligneux dans l'approvisionnement énergétique des ménages de la région restera majoritaire au cours des prochaines décennies. Sous les effets combinés de l'urbanisation et de l'accroissement démographique, la demande en boisénergie devrait continuer d'augmenter, en particulier si des sources d'énergie alternatives ne parvenaient pas à être attractives. 


\section{Tableau V.}

Production mensuelle par type de foyer amélioré et par district (année 2016).

\begin{tabular}{|c|c|c|c|c|c|c|}
\hline \multirow[t]{2}{*}{ District } & \multirow[b]{2}{*}{ Sans coque } & \multirow[b]{2}{*}{$\begin{array}{l}\text { Cylindrique } \\
\text { avec coque }\end{array}$} & \multicolumn{2}{|c|}{ Type de foyer amélioré } & \multirow[b]{2}{*}{$\begin{array}{c}\text { Carré } \\
\text { avec coque }\end{array}$} & \multirow[b]{2}{*}{ Total } \\
\hline & & & $\begin{array}{c}\text { Conique } \\
\text { avec coque }\end{array}$ & $\begin{array}{l}\text { Soupière } \\
\text { ricecooker }\end{array}$ & & \\
\hline Antananarivo Avaradrano & 400 & 0 & 0 & 0 & 400 & 800 \\
\hline Antananarivo Atsimondrano & 120 & 15970 & 2000 & 200 & 1100 & 19390 \\
\hline Total & 3520 & 17345 & 2375 & 200 & 1520 & 24960 \\
\hline
\end{tabular}

L'organisation et la modernisation de la filière bois-énergie sont par conséquent un impératif à court terme. Le nouveau cadre politique ainsi que les expériences antérieures dans le sous-secteur du bois-énergie pourraient être les ferments de cette dynamique collective portée par la région.

\section{Un cadre politique révisé à opérationnaliser}

Au cours des cinq dernières années, les autorités publiques ont révisé les politiques des secteurs de l'énergie et de l'environnement avec une considération particulière pour le bois-énergie. La Nouvelle Politique de l'énergie, promulguée en 2015, accorde une place centrale au développement de la biomasse-énergie et à l'amélioration de l'efficacité énergétique tout au long des filières, de la production à l'utilisation, en passant par la transformation et la consommation de biomasse-énergie. Elle fixe des orientations qui doivent notamment se traduire par une augmentation de l'offre légale et durable de combustibles issus de la biomasse forestière, agricole ou municipale (Gouvernement de Madagascar, 2015). La Nouvelle Politique forestière, promulguée en mai 2017 , fonde ses orientations sur la protection et la valorisation de façon durable, rationnelle et responsable des ressources forestières. Ces deux documents de politique offrent de nouvelles opportunités pour la programmation d'actions adaptées visant une gestion plus efficace et économe du bois-énergie. Cependant, la mise en œuvre de ces deux politiques est un défi majeur étant donné leurs interrelations (Gouvernement de Madagascar, 2017).

Trois documents ont été adoptés au cours de l'année 2018 afin d'encadrer cette mise en œuvre. II s'agit du Plan directeur forestier national (PDFN), des Directives nationales des actions de reboisement (DNAR) et de la Stratégie nationale d'approvisionnement en bois-énergie (SNABE). Tous les trois accordent une place importante à l'augmentation de l'offre en combustibles ligneux et à une gestion/utilisation plus efficace de la ressource. Le PDFN (MEEF, 2018a) prévoit, entre autres, l'élaboration d'une stratégie nationale de reboisement, la mise en œuvre de programmes de reboi- sement à grande échelle, la révision et la promulgation de textes de loi et d'application des incitations au reboisement, le développement des filières forestières et l'actualisation de la stratégie nationale pour le contrôle forestier. Les DNAR (MEEF, 2018b) doivent permettre d'harmoniser les actions de reboisement et favoriser la collaboration intersectorielle Énergie/Environnement. Elles articulent les objectifs autour de trois piliers, l'aménagement forestier, le foncier forestier et l'aménagement du territoire. Enfin, la SNABE (MEH, 2018) fixe trois axes stratégiques devant permettre de rétablir l'équilibre offre/demande en bois-énergie. Il s'agit de soutenir la croissance de l'offre de bois-énergie, de favoriser la réduction de la consommation de bois-énergie et enfin d'instaurer un environnement juridique favorable.

En considérant le principe de subsidiarité, la région est un premier niveau d'identification, de planification et de coordination des actions à entreprendre. Par conséquent, la validation du schéma d'approvisionnement en bois-énergie de la région a pour objectif général de favoriser la formalisation et la professionnalisation des acteurs intervenant au sein des filières bois-énergie. Un des défis à relever est de mobiliser et coordonner les différents groupes d'acteurs au niveau du district, de la commune et du fokontany afin de parvenir à la mise en œuvre effective des activités programmées.

\section{Des expériences régionales à valoriser}

La région Analamanga est une des plus importantes zones d'immigration sur la Grande Île. Le taux d'accroissement moyen pour la région est estimé à 3,9\%. Toutefois, plusieurs communes situées à proximité de la commune urbaine d'Antananarivo présentent des taux supérieurs à $5,8 \%$ et la commune rurale de Sabotsy Namehana connaît un taux d'accroissement annuel record de 13,8 \% (Région Analamanga, 2014). Les données du troisième recensement général de la population et de l'habitat, réalisé en 2018, permettront prochainement de fournir des informations plus précises sur cette concentration urbaine.

Dans ce contexte, quelles sont les voies et solutions 
pour parvenir à sécuriser l'approvisionnement en énergie des ménages tout en assurant la gestion raisonnée des espaces boisés dans lesquels l'exploitation est réalisée ?

Au cours des dix dernières années, certaines régions administratives malgaches se sont mobilisées et ont engagé des actions pour répondre à cette question. C'est notamment le cas des régions de Diana, Boeny et Atsimo Andrefana. Les stratégies qu'elles ont adoptées ainsi que les actions entreprises ont permis d’obtenir des résultats significatifs allant dans le sens d'une meilleure efficacité lors de la production et de l'utilisation de la ressource bois. Riche de ces expériences régionales, les autorités de la région Analamanga ont disposé de productions documentées (Montagne et al., 2010 ; AIDES, 2013 ; PAGE, 2015) pour engager l'élaboration du Schéma régional d'approvisionnement en bois-énergie. Certains responsables des ministères en charge de l'énergie et des forêts ont soutenu et accompagné ces initiatives régionales. Leurs expériences ont été valorisées au sein de la plateforme régionale biomasse-énergie instaurée par la Direction du développement régional Analamanga.

\section{SRABE Analamanga, une première étape pour couvrir le bassin d'approvisionnement d'Antananarivo}

Comme nous l'avons vu, les ressources forestières situées sur le territoire de la région Analamanga ne peuvent satisfaire, à elles seules, la demande de la population régionale. L'exploitation forestière réalisée dans les régions limitrophes de Alaotra Mangoro et Vakinankaratra s'accroît pour satisfaire la demande grandissante de l'agglomération d'Antananarivo. Par conséquent, il est nécessaire d'intervenir à court terme sur l'ensemble du bassin d'approvisionnement de la capitale. En s'intégrant dans le schéma institutionnel défini par la décentralisation (régions, communes) et la déconcentration (directions régionales, cantonnement au niveau du district), le SRABE Analamanga est une première étape vers une organisation des filières bois-énergie approvisionnant la capitale. La même démarche d'élaboration d'un SRABE doit être engagée dans les deux autres régions. De la sorte, elles disposeront également d'un état des lieux exhaustif sur la situation actuelle des filières bois-énergie sur leur territoire, leur permettant de prioriser et planifier les activités à mener.

Les études portant sur les flux et l'évaluation du potentiel de production de bois-énergie fournissent d'ores et déjà des données intéressantes pour argumenter la nécessité d'intervenir dans ces deux régions et identifier les études complémentaires à réaliser. La priorité est à donner à l'accompagnement de la région d'Alaotra
Mangoro qui se situe également dans le bassin versant du fleuve Mangoro, identifié comme prioritaire pour les actions de restauration des paysages et des forêts (MEEF, 2017 ; Charpin et al., 2018).

\section{Objectifs et actions principales du SRABE Analamanga}

\section{Production durable de bois-énergie}

- Harmonisation des zonages sectoriels sur le territoire communal.

- Amélioration du capital génétique des eucalyptus.

- Extension des superficies de reboisement à vocation énergétique.

- Densification/renouvellement des plantations existantes.

- Accompagnement technique des reboiseurs : techniques sylvicoles.

- Promotion et renforcement de l'intégration de l'arbre dans les systèmes agraires.

\section{Exploitation et transformation de boisénergie}

- Soutien à la formalisation des activités liées à l'exploitation (enregistrement des charbonniers, obtention des cartes professionnelles).

- Renforcement des capacités organisationnelles et entrepreneuriales des opérateurs.

- Formation des charbonniers aux techniques améliorées de carbonisation.

- Soutien à l'acquisition de fours de carbonisation semi-industriels (type GMDR).

- Promotion des briquettes agglomérées à base de fines de charbon.

\section{Transport et commercialisation du bois-énergie}

- Clarification et soutien aux initiatives des STD et CTD dans le contrôle des flux.

- Structuration d'un réseau de marchés ruraux et soutien aux circuits légaux de commercialisation.

- Communication/traçabilité sur les produits commercialisés.

- Instauration d'un système d’information sur le marché.

\section{Utilisation du bois-énergie}

- Renforcement des capacités techniques et entrepreneuriales des artisans, producteurs de foyers améliorés.

- Élargissement de la gamme des équipements de cuisson proposés aux consommateurs (y compris catégories socioprofessionnelles).

- Étude approfondie de la consommation des différentes catégories socioprofessionnelles consommatrices de boisénergie.

- Définition et mise en œuvre de stratégies de diffusion des foyers améliorés adaptées au milieu urbain et rural.

- Sécurisation et renforcement des réseaux de distribution des foyers améliorés à court terme.

- Intensification de la demande de foyers améliorés grâce à des campagnes de communication et à la densification des réseaux de distribution de proximité.

- Opérationnalisation du système de contrôle de qualité des équipements de cuisson commercialisés.

\section{Conditions cadres}

- Formulation et adoption d'un arrêté régional portant organisation de la filière boisénergie pour la région Analamanga.

- Structuration d'un dispositif de contrôle forestier efficient.

- Définition et application d'une fiscalité incitative en faveur d'une formalisation des activités.

- Analyse et applications des textes sur le foncier dans le but de sécuriser les
Figure 7.

Synthèse des actions retenues à mener par maillon. 
En référence au cadre défini par la Stratégie nationale d'approvisionnement en bois-énergie (SNABE), cinq principes guident son élaboration :

- assurer la cohérence des stratégies portant sur le bois-énergie avec les orientations nationales du développement et les politiques ou stratégies sectorielles interférant dans les processus de production et d'approvisionnement en bois-énergie ;

- garantir l'intersectorialité dans la planification, le pilotage et le suivi des actions ;

- réaffirmer les principes de la libre entreprise et de la concurrence :

- travailler dans la durée sur la base de modèles éprouvés de planification et de mise en œuvre opérationnelle ;

- participer à l'égalité des genres pour tenir compte des besoins différenciés des hommes et des femmes en matière de besoins et intérêts pour le bois-énergie, ainsi que de la féminisation de la pauvreté à Madagascar. Conformément aux axes d'intervention prioritaires de la SNABE, les objectifs spécifiques du SRABE sont au nombre de trois :

- augmenter et gérer durablement les ressources ligneuses destinées à la production de bois-énergie dans la région Analamanga en soutenant l'entrepreneuriat rural;

- maîtriser la consommation de bois-énergie grâce à une meilleure efficacité énergétique des équipements de cuisson et de combustion ;

- instaurer un environnement juridique favorable à sa mise en œuvre.

Dans ce contexte, les actions prioritaires identifiées contribueront à une production accrue et contrôlée de bois à vocation énergétique, à l'amélioration de l'efficacité énergétique des technologies de carbonisation et de combustion ainsi qu'à l'organisation et la professionnalisation des acteurs économiques intervenant sur les filières. La figure 7 présente la liste des actions devant être programmées et mises en œuvre à court et moyen terme (horizon 2030).

\section{Conclusion}

L'intérêt et la mobilisation des autorités administratives en charge de l'énergie et des forêts sont aujourd'hui explicités dans les documents de politique des deux ministères compétents. Longtemps marginalisé, le bois-énergie - et plus largement la production d'énergie à partir de la biomasse - occupe aujourd'hui une place centrale dans les orientations et les programmes sectoriels (MEEF, 2018a). Les régions, de concert avec les services techniques et les collectivités territoriales décentralisés, ont un rôle majeur à jouer pour animer le processus d'identification, de priorisation et de programmation des actions à entreprendre dans le but de rétablir l'équilibre du bilan offre/demande en bois-énergie. Dans ce contexte, la région Analamanga s'est positionnée en chef de file en animant l'élaboration du premier Schéma régional d'approvisionnement en bois-énergie
(SRABE). Les défis à relever sont nombreux pour la région la plus densément peuplée du pays et hébergeant la capitale nationale. La sécurisation de l'approvisionnement en bois-énergie de la population de l'agglomération d'Antananarivo ne pourra d'ailleurs pas être assurée par la seule région Analamanga. Une réflexion et une intervention interrégionales sont indispensables au regard de la contribution actuelle des régions de Alaotra Mangoro et Vakinankaratra à l'approvisionnement énergétique de la capitale.

Les principales actions à entreprendre dans le cadre du SRABE sont identifiées et s'inspirent des expériences réussies menées dans d'autres régions du pays. Structurées par maillon de la filière, ces actions visent à augmenter l'offre de bois-énergie en quantité et en qualité, à maîtriser la demande en bois-énergie et à instaurer les conditions cadres favorables à la mise en œuvre du SRABE. Ce dernier point concerne notamment l'instauration d'un dispositif de contrôle forestier efficient, condition indispensable à l'émergence et au développement des filières organisées et légales.

\section{Remerciements}

Les résultats présentés dans cette étude ont été obtenus dans le cadre du Programme d'appui à l'agro-sylviculture autour d'Antananarivo (ASA) financé par l'Union européenne.

Cet article se fonde sur l'expérience acquise lors de l'élaboration du Schéma régional d'approvisionnement en bois-énergie (SRABE) pour la Région Analamanga, entreprise dans le cadre d'un projet finance sous le lleme Fonds Européen de Développement pour la République de Madagascar, en étroite collaboration avec la Région Analamanga et les deux ministères techniques en charge de l'Énergie et des Forêts. Les conclusions présentées dans cet article ne reflètent pas nécessairement les opinions de la Commission européenne, et engagent la responsabilité exclusive de leurs auteurs.

\section{Références bibliographiques}

AIDES, 2013. Travaux d'inventaire et de capitalisation des cas de gouvernance de la Filière Bois Énergie au niveau de cinq régions de Madagascar : DIANA, Boeny, Atsimo Andrefana, Analamanga et Zone COFAV. Antananarivo, Madagascar, Cabinet AIDES, 116 p.

AIDES, 2018a. Étude des flux de bois-énergie entrant dans la ville d'Antananarivo. Antananarivo, Madagascar, Cabinet AIDES, 54 p. http://www.asamada.eu/les-axes-dactivitesasa/filiere-bois-energie-reboisement/

AIDES, 2018b. Étude des prix du bois-énergie (charbon de bois et bois de feu) dans la ville d'Antananarivo. Antananarivo, Madagascar, Cabinet AIDES, 34 p. http://www. asamada.eu/les-axes-dactivites-asa/filiere-bois-energie-reboisement/ 
Bertrand A., 1999. La dynamique séculaire des plantations paysannes d'eucalyptus sur les Hautes Terres malgaches. Le Flamboyant, 49 : 45-48. http://agritrop.cirad.fr/391885/

Charpin M., Rakoto Ratsimba H., Rakotovao J., Ratovoson A., Richter F., 2018. Spatialisation des engagements RPF de Madagascar dans le cadre de l'initiative AFR100. Ministère de l'Environnement, de l'Écologie et des Forêts / Deutsche Gesellschaft für Zusammenarbeit (GIZ), Madagascar, 102 p. https://afr100.org/sites/default/files/08 RPF MDG DSN Spatialisation.pdf

Gouvernement de Madagascar, 2015. Lettre de Politique de l'Énergie de Madagascar 2015-2030. Ministère de l'Énergie et des Hydrocarbures, Madagascar, 32 p. http://admin.theiguides.org/Media/Documents/LettreDePolitique.pdf

Gouvernement de Madagascar, 2017. Politique forestière de Madagascar, vers une gestion durable et responsable des forêts Malagasy. Ministère de l'Environnement, de l'Écologie et des Forêts, Madagascar, 45 p.

Land Ressources, 2018. Étude du potentiel de production en bois-énergie dans le bassin d'approvisionnement de la ville d'Antananarivo. Antsakaviro, Madagascar, Land Ressources, 36 p. http://www.asamada.eu/les-axes-dactivitesasa/filiere-bois-energie-reboisement/

LLD, 2016. Étude de marché et analyses techniques de la filière des foyers améliorés dans les zones d'intervention des Projets ARINA et AFIBERIA. Association Leadership for Local Development, Madagascar, 122 p. http://www.asamada.eu/les-axes-dactivites-asa/filiere-bois-energie-reboisement/

MEEF, 2018a. Plan directeur forestier national. Ministère de l'Environnement, de l'Écologie et des Forêts, Madagascar, $55 \mathrm{p}$.

MEEF, 2018b. Directives nationales des actions de reboisement. Ministère de l'Environnement, de l'Écologie et des Forêts, Madagascar, 105 p.

MEEF, 2017. Stratégie nationale sur la restauration des paysages forestiers et des infrastructures vertes à Madagascar. Ministère de l'Environnement, de l'Écologie et des Forêts, Madagascar, 101 p. http://www.ecologie.gov.mg/ download/strategie-nationale-sur-la-restauration-des-paysages-forestiers-et-des-infrastructures-vertes-a-madagascar-2/

MEEF-ONE, 2015. Changement de la couverture de forêts naturelles à Madagascar : 2005-2010-2013. Ministère de l'Environnement, de l'Écologie et des Forêts, Office national de l'environnement, Madagascar, 18 p. https://www.pnae. $\underline{\mathrm{mg} / \text { deforestation.html }}$

MEH, 2015. Assistance pour le développement d'une nouvelle politique énergétique et d'une stratégie pour la République de Madagascar - Phases 2 et 3. Document d'étude de la politique et stratégie énergétiques. Ministère de l'Énergie et des Hydrocarbures, Union européenne (EUEI), Madagascar, 165 p.

$\mathrm{MEH}, 2018$. Stratégie nationale d'approvisionnement en bois-énergie (SNABE). Ministère de l'Énergie et des Hydrocarbures, Madagascar, $19 \mathrm{p}$.
Montagne P., Razafimahatratra S., Rasamindisa A., Crehay R., 2010. Arina, le charbon de bois à Madagascar : entre demande urbaine et gestion durable. Antananarivo, Madagascar, CITE-Cirad, 187 p. https://www.cirad.fr/en/news/ all-news-items/articles/2012/ca-vient-de-sortir/arina-lecharbon-de-bois-a-madagascar

PAGE, 2015. Vers une modernisation de la filière bois-énergie : Série de fiches thématiques sur l'approche et les enseignements (lessons learnt) de l'expérience réalisée. Ministère de l'Environnement, de l'Écologie et des Forêts, Programme d'appui à la gestion de l'environnement (PAGE), Deutsche Gesellschaft für Internationale Zusammenarbeit (GIZ), Madagascar, 32 p. www.eco-consult.com/fileadmin/user upload/pdf/downloads/PAGE Fiches thematiques 01.pdf

Rafitoson K., 2017. La lente marche vers la transition énergétique à Madagascar : état des lieux et perspectives. Antananarivo, Madagascar, Friedrich-Ebert-Stiftung, $44 \mathrm{p}$.

Ramamonjisoa B., 1991. Approvisionnement d'Antananarivo en combustibles ligneux : mécanismes et dynamique d'évolution. Université d'Antananarivo, Madagascar, 42 p. https://infodoc.agroparistech.fr/index.php?lvl=notice display\&id=80321

Ramamonjisoa B., Rabemanajara Z., Rakoto Ratsimba H., 2016. Préparation des comptes monétaires de l'actif du bois des forêts hors Aires Protégées de Madagascar. Laboratoire de recherches appliquées, WAVES/Banque mondiale, Madagascar, 44 p. https://www.academia.edu/36070431/LA LENTE MARCHE VERS LA TRANSITION ENERGETIQUE A MADAGASCAR ETAT DES LIEUX ET PERSPECTIVES

Ramamonjisoa B. S., 1993. La ville aux mille charbonniers. ESSA, Département des Eaux et Forêts, 259 p.

Richter F., Charpin M., 2018. Modèle de simulation du bilan entre l'offre et la demande dans la Région Analamanga. Fichier sous MS Excel. Programme d'appui à l'agro-sylviculture autour d'Antananarivo, Madagascar, $12 \mathrm{p}$.

Région Analamanga, 2014. Atlas Analamanga : Compendium de 89 cartes thématiques. UN Habitat et UNDP, Madagascar, $91 \mathrm{p}$.

Sepp S., 2013. From root to soot - The wood energy value chain, elements, impacts, challenges, supporting instruments. Yaoundé, Cameroun, ECO Consulting Group, 25 p.

UPED, 1994. L'approvisionnement d'Antananarivo en combustibles ligneux. Groupement Louis Berger Sarl/SERDI, Ministère de l'Énergie et des Mines, Madagascar, $107 \mathrm{p}$

Bois et Forêts des Tropiques - Revue scientifique du Cirad
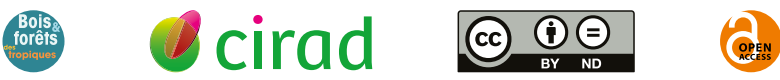

Cirad - Campus international de Baillarguet, 34398 Montpellier Cedex 5, France - Contact: bft@cirad.fr - ISSN : L-0006-579X 